\title{
Quellen und Literatur
}

\author{
I. Archivalien
}

Archiv der sozialen Demokratic, Bonn (ASD)

NL Carl Severing

NL Paul Levi

Bayerisches Hauptstaatsarchiv München (BayHStA)

MInn Ministerium des Inneren

MA Ministerium des Äußeren

G 2 Bayerische Gesandtschaft in Berlin

Bayerisches Staatsarchiv Würzburg

Landgericht Würzburg, Staatsanwaltschaft

Berlin Document Center (BDC)

Akten Pol. Präs Berlin

Personalakten

Bezirksamt Wilmersdorf von Berlin Abt. Bauwesen, Vermessungsamt

Bundesarchiv Koblenz (BA)

NS 26 NS-Hauptarchiv

R 43 I Reichskanzlei

R 58 Reichssicherheitshauptamt

NL 62 Nachlaß Harden

NL 150 Nachlaß Luctgebrune

Bundesarchiv Koblenz, Abteilungen Potsdam (BA/P)

15.07 Reichskommissar für Überwachung der öffentlichen Ordnung

30.01 Reichsjustizministerium

30.03 Oberreichsanwalt

30.09 Staatsgerichtshof zum Schutz der Republik

Deutsches Literaturarchiv, Marbach (DLitM)

NL Hermann Sudermann

Evangelisches Zentralarchiv in Berlin

1/A2 Deutscher Evangelischer Kirchenausschuß

7 Evangelischer Oberkirchenrat

Geheimes Staatsarchiv Preußischer Kulturbesitz, Berlin (GStA PK)

1. Hauptabteilung

Rep. 92 Nachlässe, NL Eugen Schiffer

Rep. 77 Ministerium des Innern

Rep. 84a Justizministerium

Hauptstaatsarchiv (HStA) Düsseldorf

Regierung Düsseldorf

Hessisches Staatsarchiv Darmstadt

Abt. G 21a Ministcrium der Justiz

Konvolut 1745 
Institut für Zeitgeschichte, München (IfZ)

Fa $163 / 1$

MA $144 / 2$

731

740

Landesarchiv Berlin

Rep. 58 Staatsanwaltschaft bei dem Landgericht Berlin

National Archives And Records Administration, Washington D.C. (NA) Microcopy T-253 NL Luetgebrune

Politisches Archiv des Auswärtigen Amts, Bonn (PA/AA)

Ref. Deutschland

Büro Reichsminister

Presse-Abteilung, Deutschland 9, Rathenau-Prozeß, Bd. 2

Sächsisches Staatsarchiv Leipzig (StAL)

Ministerium des Innern

Staatsanwaltschaft Leipzig

Sonderarchiv (jetzt: Zentrum für die Aufbewahrung der dokumentargeschichtlichen Kollektionen) Moskau (SAM)

Fonds 567 Reichsgericht und Reichsanwaltschaft

Fonds 772 Reichskommissar für Überwachung der öffentlichen Ordnung

Staatsarchiv Freiburg (StAF)

Staatsanwaltschaft Offenburg 1984/153

Staatsarchiv Hamburg (StAH)

Senat

Staatliche Pressestelle I-IV, Pol. Angelegenheiten des Deutschen Reiches, Ermordung des Reichsministers W. Rathenau, 1922-29

Staatsarchiv München

Polizeidirektion München

Staatsarchiv Schwerin

Ministerium des Innern

Stadtarchiv Gicßen

Abt. 83/92 Gießener Fememordprozeß

Universitätsarchiv Heidelberg

B 8910 Disziplinarangelegenheiten, Einzelfälle 


\section{Nachlässe und Sammlungen in privater Hand}

NL Edith Andreae, Zürich

NL Hartmut Plaas, Memmingen

NL Ernst von Salomon, Stöckte über Winsen a. d. Luhe

Sammlung Hans Prescher, Frankfurt a. M.

\section{Dokumentationen, Quelleneditionen, Hilfsmittel}

Akten der Reichskanzlei, Weimarer Republik, hrsg. für die Historische Kommission der Bayerischen Akademie der Wissenschaften von Karl Dietrich Erdmann, für das Bundesarchiv von Hans Booms: Die Kabinette Wirth I und II, 10. Mai 1921 bis 26. Oktober 1921, 26. Oktober 1921 bis 22. November 1922, bearb. von Ingrid Schulze-Bidlingmaier, Boppard 1973.

Akten zur deutschen auswärtigen Politik 1918-1945, Serie A: 1918-1925, Band 6, 1. März bis 31. Dezember 1922, Göttingen 1988.

Benz, Wolfgang (Hrsg.): Politik in Bayern 1919-1933. Berichte des württembergischen Gesandten Carl Moser von Filseck, Stuttgart 1971.

Brammer, Karl: Attentäter, Spitzel und Justizrat Claß. Der Seeckt-Harden-Prozeß, Berlin 1924.

Ders.: Das politische Ergebnis des Rathenau-Prozesses, Berlin 1922.

Deuerlein, Ernst (Hrsg.): Der Aufstieg der NSDAP in Augenzeugenberichten, München 1974.

Ders. (Hrsg.): Der Hitler-Putsch. Bayerische Dokumente zum 8./9. November 1923, Stuttgart 1962.

Der Erzberger-Mord. Dokumente menschlicher und politischer Verkommenheit, Brühl 1921.

Gegen den politischen Mord! Reichstagssitzung vom 25. Juni 1922, Berlin 1922.

Gottlieb, Ernst: Walther-Rathenau-Bibliographie, Berlin 1929.

Hellige, Hans Dieter (Hrsg.): Walther Rathenau/Maximilian Harden. Briefwechsel 1897-1920, München/Heidelberg 1983.

Horkenbach, Cuno (Hrsg.): Das Deutsche Reich von 1918 bis heute, Berlin 1930.

Jasper, Gotthard: Aus den Akten der Prozesse gegen die Erzberger-Mörder, in: VfZ 10 (1962), S. 430453.

Morsey, Rudolf/Ruppert, Carsten (Bearb.): Die Protokolle der Reichstagsfraktion der Deutschen Zentrumspartei 1920-1925, Mainz 1981.

Pfeiffer, A. Ch.: Das Welt-Echo des Rathenau-Mordes. Stimmen und Urteile des Auslandes über Deutschland aus Anlaß der Ermordung Rathenaus, Berlin 1922.

Pfeiffer-Belli, Wolfgang (Hrsg.): Harry Graf Kessler. Tagebücher 1918-1937, Frankfurt a. M. 1961.

Pogge-von Strandmann, Hartmut (Hrsg.): Walther Rathenau. Tagebuch 1907-1922, Düsseldorf 1967.

Reichsgesetzblatt $1921 \mathrm{ff}$., Teil 1, hrsg. vom Reichsministerium des Innern, Berlin $1921 \mathrm{ff}$.

Schulin, Ernst (Hrsg.): Walther Rathenau. Hauptwerke und Gespräche, München/Heidelberg 1977.

Schulthess' Europäischer Geschichtskalender, Neue Folge, Jg. 1921 und 1922, München 1927.

Schweder, Paul: Der Erzberger-Mord-Prozeß. Vorgeschichte, Anklageschrift und ausführlicher Verhandlungsbericht, Rudolstadt/Thür. o. J. [1921].

Straßenverzeichnis von Wilmersdorf, hrsg. vom Bezirksamt Wilmersdorf, Berlin o. J.

Tyrell, Albrecht (Hrsg.): Führer befiehl ... Selbstzeugnisse aus der, Kampfzeit' der NSDAP. Dokumentation und Analyse, Düsseldorf 1969.

Verhandlungen des deutschen Reichstages, 1. Wahlperiode. Stenographische Berichte und Anlagen, Bde. 346-359, Berlin $1920 \mathrm{ff}$.

Verhandlungen des Sächsischen Landtages, 1. Sitzungsperiode, 113. Sitzung, Leipzig $1919 \mathrm{ff}$.

Wcrthauer, Johannes: Das Blausäure-Attentat auf Scheidemann. Aktenmäßige Darstellung auf Grund der Verhandlung vor dem Staatsgerichtshof, Berlin 1923.

Wirth, Joseph: Reden während der Kanzlerschaft, Berlin 1925. 


\section{Memoiren, literarische Darstellungen, zeitgenössische Zeugnisse}

Anonymus: Ein Beitrag zur Judenstatistik, in: Allgemeine Zeitung des Judentums, 22. 12. 1916.

Anonymus: Genua, in: Der Wiking vom 15. 3. 1922.

Anonymus: Genua. III., in: Der Wiking vom 1.6.1922.

Anonymus: Minister Rathenau, in: Der Wiking vom 15. 2. 1922.

Anonymus: Philipp Lenard. Der deutsche Naturforscher, München/Berlin 1937.

Viscount d' Abernon, Edgar Vincent: Ein Botschafter der Zeitenwende, 3 Bde., Leipzig o. J.

Ahrens, Georg: Erinnerungen an Walther Rathenau. Zur Wiederkehr seines Todestages am 24. Juni 1922, in: „Die Alte Schule“. Mitteilungen der Vereinigung ehemaliger Schüler der Walther-RathenauSchule - Grunewald-Gymnasium, Nr. 58, 1962, S. 2-5.

Alsberg, Max: Der Rathenau-Prozeß. Ein juristischer Epilog, in: Deutsche Juristen-Zeitung 1922, H. 21/ 22, Sp. 663-666.

Beek, Gottfried zur [Müller von Hausen, Ludwig] (Hrsg.): Die Geheimnisse der Weisen von Zion, Charlottenburg 1919.

Bernhard, Georg: Der Fall Rathenau, in: Vossische Zeitung vom 4. 7. 1920.

Blei, Franz: Männer und Masken, Berlin 1930.

Blome, Kurt: Arzt im Kampf. Erlebnisse und Gedanken, Leipzig 1942.

Blumenfeld, Kurt: Erlebte Judenfrage. Ein Vierteljahrhundert deutscher Zionismus, Stuttgart 1962.

Boveri, Margret: Verzweigungen. Eine Autobiographie, hrsg. von Uwe Johnson, München 1982.

Brandt, Rolf: So sieht die Weltgeschichte aus ... Aufzeichnungen eines Zuschauers, Hamburg 1926.

Braun, Otto: Von Weimar zu Hitler, Hamburg 1949.

Brecht, Arnold: Aus nächster Nähe. Lebenserinnerungen 1884-1927, Stuttgart 1966.

Ders.: Vorspiel zum Schweigen. Das Ende der deutschen Republik, Wien 1948.

Ders.: Walther Rathenau und das deutsche Volk, München 1950.

Curator, Carsten: Putsche, Staat und wir, Karlsruhe 1931.

Deutschvölkischer Katechismus, hrsg. von einem deutschen Hochschullehrer, Leipzig 1931.

Ebermayer, Ludwig: Fünfzig Jahre Dienst am Recht. Erinnerungen eines Juristen, Leipzig/Zürich 1930.

Eggebrecht, Axel: Der halbe Weg, Reinbek bei Hamburg 1981.

Ehrhardt, Hermann: Deutschlands Zukunft, Aufgaben und Ziele, München 1921.

F., O.: Suche Brot in der Fremde, in: Deutsche Tageszeitung vom 2. 2. 1919.

Ders.: Walther Rathenau und Kaiser Wilhelm II, in: Deutsche Tageszeitung vom 14. 4. 1919.

Faßland, Frank [Pinner, Felix]: Wirtschafts-Führer, I. Walther Rathenau, in: Die Weltbühne vom 23. 2. 1922, S. $185-191$.

Frank, Walter: „Höre Isreal!“. Studien zur modernen Judenfrage, Hamburg ${ }^{2} 1942$.

Freksa, Friedrich (Hrsg.): Kapitän Ehrhardt. Abenteuer und Schicksale. Nacherzählt von ***, Berlin 1924.

Fürstenberg, Carl: Die Lebensgeschichte eines deutschen Bankiers, niedergeschrieben von Hans Fürstenberg, Wiesbaden o. J. [1961].

Fürstenberg, Hans: Erinnerung an Walther Rathenau, in: Kessler, Walther Rathenau, S. 373-447.

Ganz, Hans: Industriekapitäne, II. Walther Rathenau, in: Die Weltbühne vom 17. 6. 1920, S. 718 ff.

Gellert, Wilhelm: Der Zusammenbruch der Demokratie. Zum Tode Rathenaus. Sozialisten und Demokraten im Spiegel ihrer Worte und Taten, Berlin 1922.

Gerlach, Hellmut von: Von rechts nach links, Zürich 1937.

Goldschmidt, Alfons: Retter Rathenau, in: Die Weltbühne vom 17. 10. 1918, S. $372 \mathrm{ff.}$

Großmann, Stefan: Walther Rathenau, in: Das Tage-Buch vom 11. 6. 1921, S. 708-711.

Gumbel, Emil Julius: „Verräter verfallen der Feme“. Opfer/Mörder/Richter 1919-1929. Unter Mitwirkung von Berthold Jacob und Ernst Falck, Berlin 1929.

Ders.: Verschwörer. Zur Geschichte und Soziologie der deutschen nationalistischen Geheimbünde 1918-1924, Neuausg. Frankfurt a. M. 1984.

Ders.: Vom Fememord zur Reichskanzlei. Mit einem Vorwort von Walter Fabian, Heidelberg 1962.

Haase, Ernst (Hrsg.): Hugo Haase. Sein Leben und Wirken, Berlin o. J.

Haber, Charlotte: Mein Leben mit Fritz Haber.Spiegelungen derVergangenheit, Düsseldorf/Wien 1970.

Hachenberg, Max: Juristische Rundschau, in: Deutsche Juristen-Zeitung 1922, H. 15/16, Sp. 492-497.

Ders.: Juristische Rundschau, in: Deutsche Juristen-Zeitung 1922, H. 21/22, Sp. 669-674.

Harden, Maximilian: In der Mördergrube, in: Die Zukunft vom 1. 7. 1922, S. 1-6.

Ders.: Lüge in Schneeweiß, in: Die Zukunft vom 11. 2. 1922, S. 145-177. 
Ders.: Rausch aus Hurenwein, in: Die Zukunft vom 4. 2. 1922, S. 115-144.

Ders.: Die Riesendummheit, in: Die Zukunft vom 22. 4. 1922, S. 85-106.

Ders.: Sexagesima, in: Die Zukunft vom 18. 2. 1922, S. 175-205.

Ders.: Tönt der Glocke Grabgesang?, in: Die Zukunft vom 29. 7./5. 8 1922, S. 56-80.

Ders.: Zum Schutz der Republik. Techow \& Söhne, in: Die Zukunft vom 8./22. 7. 122, S. 27-32.

Heinz, Friedrich Wilhelm: Ministermorde, in: Wulf Bley (Hrsg.), Revolutionen der Weltgeschichte.

Zwei Jahrtausende Revolutionen und Bürgerkriege, München 1933, S. 839-851.

Ders.: Die Nation greift an. Geschichte und Kritik des soldatischen Nationalismus, Berlin 1933.

Ders.: Politische Attentate in Deutschland, in: Hotzel (Hrsg.), Deutscher Aufstand, S. 190-210.

Ders.: Sprengstoff, Berlin 1930.

Helfferich, Karl: Fort mit Erzberger, Berlin 1919.

Heuß, Theodor: Erinnerungen 1905-1933, Tübingen 1963.

Hitler, Adolf: Mein Kampf, München ${ }^{748-7521942 .}$

Hottenrott, Hans: Offener Brief an Herrn Dr. Walther Rathenau, in: Mitteldeutsche Presse vom 1.3. 1922.

Hotzel, Curt (Hrsg.): Deutscher Aufstand. Die Revolution des Nachkriegs, Stuttgart 1934.

Keil, Wilhelm: Erlebnisse eines Sozialdemokraten, 2 Bde., Stuttgart 1948.

Kerr, Alfred: Walther Rathenau, in: Pan vom 19. 9. 1912.

Ders.: Walther Rathenau. Erinnerungen eines Freundes, Amstcrdam 1935.

Kessler, Harry Graf/Ludwig, Emil/Mann, Heinrich u. a.: Maximilian Harden zum 20. Oktober 1921. 1861-1921, Berlin 1921.

Krebs, Albert: Tendenzen und Gestalten der NSDAP. Erinnerungen an die Frühzeit der Partei, Stuttgart 1959.

Lambach, Walther: Diktator Rathenau, Hamburg/Leipzig, ${ }^{2} 1918$.

Lania, Leo [Hermann, Lazar]: Die Organisation Consul, in: Die Weltbühne vom 30. 10.1924, S. 655-658.

Lemmer, Ernst: Manches war doch anders, Frankfurt a. M. 1968.

Lessing, Theodor: Der jüdische Selbsthaß, München 1984 (Nachdruck der Ausgabe Berlin 1930).

Löbe, Paul: Der Weg war lang. Lebenserinnerungen von Paul Löbe, Berlin ${ }^{2} 1954$.

Mann, Thomas: Von Deutscher Republik. Rede, gehalten am 15. 10. 1922 in Berlin, in: Ders., Politische Schriften und Reden, S. 100-130.

Ders.: Geist und Wesen der Deutschen Republik. Dem Gedächtnis Walther Rathenaus, in: Ders., Politische Schriften und Reden, S. 132-138.

Ders.: Politische Schriften und Reden, hrsg. von Hans Bürgin, Bd. 2, Frankfurt a. M./Hamburg 1968.

Marx, Hugo. Werdegang eines jüdischen Staatsanwalts und Richters in Baden (1892-1933). Ein soziologisch-politisches Zeitbild, Villingen 1965.

Mayer, Eugen: Skizzen aus dem Leben der Weimarer Republik. Berliner Erinnerungen, Berlin 1962.

Meinecke, Friedrich: Der Geist der akademischen Jugend in Deutschland. Zur Erklärung der psychologischen Ursachen des Rathenau-Mordes, in: Ders., Politische Schriften und Reden, hrsg. und eingel. von Georg Kotowski, Darmstadt 1958, S. 338-343.

Meyer-Leviné, Rosa: Im inneren Kreis. Erinnerungen einer Kommunistin in Deutschland 1920-1933, Köln 1979.

Morus [Lewinsohn, Richard]: Das Ergebnis von Genua, in: Die Weltbühne vom 25. 5. 1922, S. $534 \mathrm{ff}$.

Ders.: Katastrophenpolitik. Der Vertrag von Rapallo, in: Die Weltbühne vom 27. 4. 1922, S. 427 ff.

Norlind, Ernst: Gespräche und Briefe Walther Rathenaus, Dresden o. J.

Oestreich, Paul: Rathenaus Kaiser-Epilog, in: Die Tat, August 1919, S. 394 ff.

Olf [Leonhard, Rudolf]: Die Reaktion, in: Die Weltbühne vom 28. 11. 1918, S. 499 f.

Plaas, Hartmut (Hrsg.): Wir klagen an! Nationalisten in den Kerkern der Bourgeoisie, Berlin 1928.

Pinner, Felix: Diktatur der Schwerindustrie, in: Berliner Tageblatt vom 15. 5. 1920.

Poelchau, Harald: Die Ordnung der Bedrängten, Berlin 1963.

Radbruch, Gustav: Der innere Weg. Aufriß meines Lebens, Göttingen ${ }^{2} 1961$.

Rathenau, Walther: Briefe, 2 Bde., Dresden 1926/27.

Ders.: Ein dunkler Tag, in: Vossische Zeitung vom 7. 10. 1918.

Ders.: Impressionen, Berlin 1902.

Ders.: Der Kaiser, in: Ders., Schriften aus Kriegs- und Nachkriegszeit, S. 283-338.

Ders.: Kritik der dreifachen Revolution. Apologie, Berlin 1919.

Ders.: Neue Briefe, Dresden 1927.

Ders.: Politische Briefe, Dresden 1929.

Ders.: Reflexionen, Berlin 1908.

Ders.: Schriften aus Kriegs- und Nachkriegszeit, Berlin 1929. 
Ders.: Staat und Judentum. Eine Polemik, in: Ders., Gesammelte Schriften, Bd. 1, Berlin 1925, S. 183-207.

Ders.: Unser Nachwuchs, in: Neue Freie Presse, Wien, 25. 12. 1909.

Ders.: Von kommenden Dingen (Gesammelte Schriften, Bd. 3), Berlin 1925.

Ders.: Was wird werden, Berlin 1920.

Ders.: Zur Mechanik des Geistes oder Vom Reich der Seele (Gesammelte Schriften, Bd. 2), Berlin 1925.

Redslob, Edwin: Von Weimar nach Europa. Erlebtes und Durchdachtes, Berlin 1972.

Reichert, Jakob Wilhelm: Rathenausche Zwangswirtschaft, in: Stahl und Eisen vom 28. 7. 1921, S. 10301037.

Reventlow, Ernst zu: Herr Dr. Rathenau im Wirtschaftsrat, in: Deutsche Tageszeitung vom 30. 7. 1920.

Roddie, Stewart: Peace Patrol, London 1932.

Roden, Hans: Widerstand, Leipzig 1938.

Roderich-Stoltheim, Ferdinand [Fritsch, Theodor]: Anti-Rathenau, Leipzig 1919.

Röhm, Ernst: Die Geschichte eines Hochverräters, München ${ }^{6} 1934$.

Rosenberg, Alfred: „Neue Zeitung“ gegen „Neuc Zeitung“, in: Völkischer Beobachter vom 8. 2. 1922.

Roth, Alfred: Rathenau. „Der Kandidat des Auslandes“, Hamburg 1922.

Roth, Joseph: Berliner Saisonbericht. Unbekannte Reportagen und journalistische Arbeiten 1920-1939, hrsg. von Klaus Westermann, Köln 1984.

S., W.: Hans Dampf in allen Gassen, in: Deutsche Tageszeitung vom 9. 7. 1921.

Salomon, Ernst von: „Verschwörer“, in: Das Reich im Werden. Arbeitshefte im Dienste politischer Erziehung, hrsg. von Rudolf Ibel, Frankfurt 1933.

Ders.: Der Fragebogen, Hamburg 1951.

Dcrs: Dic Geächteten, Gütersloh ${ }^{8} \mathrm{o}$. J.

Scheidemann, Philipp: Zwischen den Gefechten, Berlin 1920.

Ders.: Memoiren eines Sozialdemokraten, 2 Bde., Dresden 1928.

Ders.: Die rechtsradikalen Verschwörer, Berlin 1923.

Ders.: Der Zusammenbruch, Berlin 1921.

Schiffer, Eugen: Ein Leben für den Liberalismus, Berlin 1951.

Schwaner, Wilm: Walther Rathenau, in: Der Volkserzieher vom 5. 8. 1922.

Schweyer, Franz: Politische Geheimverbände. Blicke in die Vergangenheit und Gegenwart des Geheimbundwesens, Frciburg 1925.

Severing, Carl: Mein Lcbensweg, 2 Bde., Köln 1950.

Simon, Hugo-Ferdinand: Aus Walther Rathenaus Leben, Dresden 1927.

Spiero, Heinrich: Schicksal und Anteil. Ein Lebensweg in deutscher Wendezeit, Berlin 1929.

Stein, Hans Wilhelm: Burg Saaleck. Die Türme des Schweigens, Eckartsberga/Thür. o. J.

Stockhausen, Max von: Sechs Jahre Reichskanzlei. Von Rapallo bis Locarno. Erinnerungen und Tagebuchnotizen 1922-1927, bearb. und hrsg. von Walter Görlitz, Bonn 1954.

Techow, Ernst-Werner: „Gemeiner Mördcr?!“ Das Rathenau-Attentat, Leipzig o. J.

Techow, Hans-Gerd: Minister Rathenaus Ende, in: Der Angriff vom 22. 10., 29. 10., 5. 11., 19. 11., 26. 11., 3. 12. 1928.

Thimme, W.: Rathenau als Publizist, in: Deutsche Politik vom 3. 5. 1918, S. 565-570.

Troeltsch, Ernst: Dem crmordeten Freunde, in: Ders., Deutscher Geist und Westeuropa, hrsg. von Hans Baron, Tübingen 1925.

Ders.: Spektator-Briefe. Aufsätze über die deutsche Revolution und die Weltpolitik 1928-1922, hrsg. von Hans Baron, Tübingen 1924.

Tucholsky, Kurt: Der Schnellmaler, in: Die Weltbühne vom 29. 5. 1919, S. 616-619.

Ders.: Prozeß Harden, in: Die Weltbühne vom 21. 12. 1922, S. 638-645.

Ders.: Was wäre, wenn ...?, in: Die Weltbühne vom 22. 6. 1922, S. 615-620.

Tyrus: Brief an Herrn Rathenau, in: Die Weltbühne vom 9. 2. 1922, S. 131-134.

W., H.: Rathenaus Stellung zur Judenfragc, in: Neue Preußische Zcitung - Kreuzzeitung vom 30.6. 1921.

Warburg, Max M.: Aus meinen Aufzeichnungen, Privatdruck New York 1952.

Weiß, Bernhard: Polizei und Politik, Berlin 1928.

Wendt, Hans: Journalist im Krisenreichstag. Das parlamentarische System der Weimarer Republik, in: Rudolf Pörtner (Hrsg.), Alltag in der Weimarer Republik. Erinnerungen an eine unruhige Zeit, Düsseldorf/Wien/New York 1990, S. 68-95.

Westarp, Kuno Graf von: Die innere Politik der Woche, in: Neue Preußische Zeitung - Kreuzzeitung vom 5. 6. 1921.

Wicse, Leopold von: Freie Wirtschaft, Leipzig 1918.

Wirth, Joseph: Walther Rathenau vor seinem Tode, in: Deutsche Republik vom 13. 7. 1928, S.1305-1308. 
Zechlin, Walter: Pressechef bei Ebert, Hindenburg und Kopf, Hannover 1956.

Zeiler, Alois: Meine Mitarbeit, o. O. u. o. J. [1938].

Zuckmayer, Carl: Carlo Mierendorff. Porträt eines deutschen Sozialisten, Berlin 1947.

Zweig, Stefan: Die Welt von gestern. Erinnerungen eines Europäers, Gütersloh 1960.

\section{Sekundärliteratur}

Ackermann, Volker: Nationale Totenfeiern in Deutschland. Von Wilhelm I. bis Franz Josef Strauß, Stuttgart 1990.

Angress, Werner T.: Die Kampfzeit der KPD 1921-1923, dt. Ausg. Düsseldorf 1973.

Battenberg, Friedrich: Das Europäische Zeitalter der Juden. Zur Entwicklung einer Minderheit in der nichtjüdischen Umwelt Europas, 2 Teilbde., Darmstadt 1990.

Baumann, Udo/Gebauer, Gertrude: Zur politischen Geschichte der Universität Leipzig 1921-1923, in: Karl-Marx-Universität Leipzig 1409-1959. Beiträge zur Universitätsgeschichte, 2. Band, Leipzig 1959, S. 120-155.

Bennecke, Heinrich: Hitler und die SA, München/Wien 1962.

Berding, Helmut: Moderner Antisemitismus in Deutschland, Frankfurt a. M. 1988.

Berghahn, Volker R.: Der Stahlhelm. Bund der Frontsoldaten 1918-1935, Düsseldorf 1966.

Berglar, Peter: Walther Rathenau. Fin L eben zwischen Philosophie und Politik, Graz/Wien/Köln 1987.

Bering, Dietz: Kampf um Namen. Bernhard Weiß gegen Joseph Goebbels, Stuttgart ${ }^{2} 1992$.

Bernett, Hajo: Völkische Turner als politische Terroristen, in: Sportwissenschaft 4 (1992), S. 418-439.

Beyerchen, Alan D.: Wissenschaftler unter Hitler. Physiker im Dritten Reich, Köln 1980.

Bleuel, Hans Peter/Klinnert, Ernst: Deutsche Studenten auf dem Weg ins Dritte Reich. Ideologien Programme - Aktionen 1918-1935, Gütersloh 1967.

Boveri, Margret: Der Verrat im 20. Jahrhundert, II.: Für und gegen die Nation. Das unsichtbare Geschehen, Hamburg 1956.

Breger, Herbert: Streifzug durch die Geschichte der Mathematik und Physik an der Universität Heidelberg, in: Karin Buselmeier/Dietrich Harth/Christian Jansen(Hrsg.), Auch eine Geschichte der Universität Heidelberg, Mannheim 1985, S. 27-50.

Buddensieg, Tilmann/Hughes, Thomas/Kocka, Jürgen u. a.: Ein Mann vieler Eigenschaften. Walther Rathenau und die Kultur der Moderne, Berlin 1990.

Buder, Johannes: Die Reorganisation der preußischen Polizei 1918-1923, Frankfurt a. M./Bern/New York 1986.

Carlé, Wilhelm: Weltanschauung und Presse. Eine soziologische Untersuchung, Leipzig 1931.

Carsten, Francis L.: Reichswehr und Politik 1918-1933, Köln/Berlin 1964.

Diehl, James M.: Paramilitary Politics in Weimar Germany, Bloomington/London 1977.

Eimers, Enno: Das Verhältnis von Preußen und Reich in den ersten Jahren der Weimarer Republik, Berlin 1969.

Epstein, Klaus: Matthias Erzberger und das Dilemma der deutschen Demokratie, Frankfurt a. M./Berlin 1962.

Erdmann, Karl Dietrich: Die Geschichte der Weimarer Republik als Problem der Wissenschaft, in: VfZ 3 (1955), S. 1-19.

Ders.: Die Weimarer Republik, München 1980.

Erman, Hans: Berliner Geschichten. Geschichte Berlins. Historien. Episoden. Anekdoten, Tübingen ${ }^{3} 1967$.

Exiner, Robert: Erwin Kern - A Study of Political Violence in Germany 1918-1922, Melbourne (MS) 1982.

Federn-Kohlhaas, Etta: Walther Rathenau. Sein Leben und Wirken, Dresden 1927.

Feldman, Gerald D.: Der unschlüssige Staatsmann. Rathenaus letzter Tag und die Krise der Weimarer Republik, in: Buddensieg/Hughes/Kocka u. a., Ein Mann vieler Eigenschaften, S. 84-98.

Fenske, Hans: Konservativismus und Rechtsradikalismus in Bayern nach 1918, Bad Homburg/Berlin/ Zürich 1969.

Finker, Kurt: Bund Wiking (BW) 1923-1928, in: Dieter Fricke/Werner Fritsch/Herbert Gottwald u. a. (Hrsg.), Lexikon zur Parteiengeschichte. Die bürgerlichen und kleinbürgerlichen Parteien und Verbände in Deutschland (1789-1945), Bd. 1, Leipzig 1983, S. 368-373.

Ders.: Die militärischen Wehrverbände in der Weimarer Republik. Ein Beitrag zur Strategie und Taktik der deutschen Großbourgeoisie, in: ZfG 14 (1966), S. 357-377. 
Flade, Roland: „Es kann sein, daß wir eine Diktatur brauchen“. Rechtsradikalismus und Demokratiefeindschaft in der Weimarer Republik am Beispiel Würzburg, Würzburg 1983.

Ford, Franklin L.: Der politische Mord. Von der Antike bis zur Gegenwart, Hamburg 1991.

Giovannini, Norbert: Zwischen Republik und Faschismus: Heidelberger Studentinnen und Studenten 1918-1945, Weinheim 1990.

Giraud, Pierre: L'image de Walther Rathenau dans la presse et la litterature allemandes. Sa légende historique, ses métamorphoses litteraires, Diss. Paris 1975.

Goldscheider, G.: Heinrich Tillessen und seine Welt, in: Frankfurter Hefte 1947, S. 349-357.

Goodrick-Clarke, Nicholas: The Occult Roots of Nazism. Secret Aryan Cults and Their Influence on Nazi Idcology. The Ariosophists of Austria and Germany 1890-1935, London ${ }^{2} 1992$.

Gordon jr., Harald J.: Hitler-Putsch 1923. Machtkampf in Bayern 1923-1924, Frankfurt a. M. 1971.

Griewank, Karl: Dr. Wirth und die Krisen der Weimarer Republik, in: Wissenschaftliche Zeitschrift der Universität Jena, 1951/52, H. 2, S. 1-10.

Grözinger, Wolfgang: Walther Rathenau 1867-1922, in: Deutsche Demokratie von Bebel bis Heuß. Geschichte in Lebensbildern, hrsg. von Friedrich Michael/Sybil Gräfin Schönfeldt, Hamburg 1964, S. 77-94.

Haffner, Scbastian: Schüsse von rechts, in: Stern vom 22. 6. 1972.

Hamilton, Alastair: L'illusion fasciste. Les intellectuels et le fascime. 1919-1945, Paris 1971.

Hammer, Erich: Gustav Techow und der Zeughaussturm am 14. Juni1848 und die ferneren Schicksale des Techow, in: Zeitschrift des Vereins für die Geschichte Berlins, 1938, S. 52-57.

Hannover, Heinrich/Hannover-Drück, Elisabeth: Politische Justiz 1918-1933. Mit einem Vorwort von Joachim Perels, Bornheim-Merten 1987.

Hecker, Gerhard: Walther Rathenau und sein Verhältnis zu Militär und Krieg, Boppard am Rhein 1983.

Helfferich, Philipp: Rathenau und Helfferich. Brief an die Herausgeber, in: Die Gegenwart 2 (1947), H. 15/16, S. 24.

Hellige, Hans Dieter: Generationskonflikt, Selbsthaß und die Entstehung antikapitalistischer Positionen im Judentum. Der Einfluß des Antisemitismus auf das Sozialverhalten jüdischer Kaufmanns- und Unternehmersöhne im Deutschen Kaiserreich und in der K.u.K.-Monarchic, in: Geschichte und Gesellschaft 5 (1979), S. 476-518.

Ders.: Rathenau und Harden in der Gesellschaft des Deutschen Kaiserreichs. Eine sozialgeschichtlichbiographische Studie zur Entstehung neokonservativer Positionen bei Unternehmern und Intellektucllen, in: Hellige (Hrsg.), Rathenau/Harden. Briefwechsel 1897-1920, S. 15-299.

Henke, Josef: Das Schicksal deutscher zeitgeschichtlicher Quellen in Kriegs- und Nachkriegszeit, in: VfZ 30 (1982), S. 557-620.

Heydeloff, Rudolf: Staranwalt der Rechtsextremisten. Walter Luctgebrune in der Weimarer Republik, in: VfZ 32 (1984), S. 373-421.

Ders.: The Political-Judicial Career of Dr. jur. Walter Luetgebrune and the Crisis of Weimar and Early National Socialist Germany: 1918 to 1934, Diss. Waterloo/Ontario 1976.

Hoegner, Wilhelm: Der politische Radikalismus in Deutschland 1919-1933, München/Wien 1966.

Ders.: Die verratene Republik. Deutsche Geschichte 1919-1933, Neuausg. München 1979.

Höhne, Heinz: Canaris. Patriot im Zwielicht, Gütersloh 1984.

Hoffmann, Peter: Widerstand, Staatsstreich, Attentat. Der Kampf der Opposition gegen Hitler, München ${ }^{4} 1985$.

Hofmann, Hans-Hubert: Der Hitler-Putsch. Krisenjahre deutscher Geschichte 1920 bis 1924, München 1961.

Huber, Ernst Rudolf: Deutsche Verfassungsgeschichte seit 1789, Bd. 7: Ausbau, Schutz und Untergang der Weimarer Republik, Stuttgart/Berlin/Köln u. a. 1984.

Jansen, Christian: Professoren und Politik. Politisches Denken und Handeln der Heidelberger Hochschullehrer 1914-1935, Göttingen 1992.

Jasper, Gotthard: Der Schutz der Republik. Studien zur staatlichen Sicherung der Demokratie in der Weimarer Republik 1922-1930, Tübingen 1963.

Ders.: Justiz und Politik in der Weimarer Republik, in: VfZ 30 (1982), S. 167-205.

Jatho, Jörg-Peter: Vorfaschismus in Gießen 1890-1933, Gießen 1988.

Jena, Kai von/Lenz, Wilhelm: Die deutschen Bestände im Sonderarchiv in Moskau, in: Der Archivar 45 (1992), S. 457-468.

Kallner, Rudolf: Herzl und Rathenau. Wege jüdischer Existenz an der Wende des 20. Jahrhunderts, Stuttgart 1976.

Kessler, Harry Graf: Walther Rathenau. Sein Leben und sein Werk. Mit einem Kommentar von Hans Fürstenberg, Wiesbaden o. J. (1962) (Neuausg. hrsg. von Cornelia Blasberg/Gcrhard Schuster, Frankfurt a. M. 1988). 
Klass, Gert von: Hugo Stinnes, Tübingen 1958.

Kleinhans, Ludger: Ein bemerkenswerter Fund auf dem Dachboden des Pfarrhauses, in: Wegspuren. Fünfzig Jahre Herz-Jesu-Kirche zu Rheine/Westf., hrsg. von der Gemeinde „Herz-Jesu“, Rheine 1988, S. 41-44.

Knauß, Erwin: Der Gießener Fememordprozeß von 1927, in: Archiv für hessische Geschichte und Altertumskunde, Neue Folge, 32. Bd., Darmstadt 1974, S. 111-158.

Koch, Hannsjoachim W.: Der deutsche Bürgerkrieg. Eine Geschichte der deutschen und österreichischen Freikorps 1918-1923, Berlin/Frankfurt a. M./Wien 1978.

Kreutzberger, Wolfgang: Studenten und Politik 1918-1933. Der Fall Freiburg im Breisgau, Göttingen 1972.

Krüger, Gabriele: Die Brigade Ehrhardt, Hamburg 1971.

Kruppa, Bernd: Rechtsradikalismus in Berlin 1918-1922, Berlin/New York 1988.

Krusch, Hans-Joachim: Der Kampf der Zwickauer Werktätigen während der Protestbewegung gegen den Mord an Walther Rathenau für die Bändigung des deutschen Militarismus und eine demokratische Außenpolitik, in: Sächsische Heimatblätter 7 (1961), S. 222-228.

Lamm, Hans: Walther Rathenau. Denker und Staatsmann, Hannover 1968.

Lange, Günther: Die Protestaktionen der Zwickauer Arbeiter gegen den deutschen Militarismus aus Anlaß des Mordes an Walter Rathenau im Sommer 1922, in: Beiträge zur Geschichte der deutschen Arbeiterbewegung 4 (1962), S. 950-964.

Langemann, Hans: Das Attentat. Eine kriminalwissenschaftliche Studie zum politischen Kapitalverbrechen, Hamburg 1956.

Laubach, Ernst: Die Politik der Kabinette Wirth 1921/22, in: Historische Studien, Heft 402, Lübeck/ Hamburg 1968.

Létourneau, Paul: Walther Rathenau ou le rêve prométhéen (1867-1922), Québec 1987.

Loewenberg, Peter: "The Murder and Mystification of Walther Rathenau", Los Angeles (MS) 1991.

Lohalm, Uwe: Völkischer Radikalismus. Die Geschichte des Deutschvölkischen Schutz- und Trutzbundes 1919-1923, Hamburg 1970.

Mader, Ursula: Wie das „Deutschlandlied“ 1922 Nationalhymne wurde. Aus der Ministerialakte „Nationallied“, in: ZfG 38 (1990), S. 1088-1100.

Mahlke, Bernhard: Organisation Consul (OC) 1920-1923, in: Dieter Fricke/Manfred Weißbecker/ Herbert Schwab u. a. (Hrsg.), Die bürgerlichen Parteien in Deutschland. Handbuch der Geschichte der bürgerlichen Parteien und anderer bürgerlicher Interessenorganisationen vom Vormärz bis zum Jahre 1945, Bd. 2, Berlin (O) 1970, S. 454-458.

Mann, Golo: Deutsche Geschichte des 19. und 20. Jahrhunderts, Frankfurt a. M. 1958.

Maser, Werner: Der Sturm auf die Republik. Frühgeschichte der NSDAP, Frankfurt a. M./Berlin/Wien 1981.

Mauch, Hans-Joachim: Nationalistische Wehrorganisationen in der Weimarer Republik. Zur Entwicklung und Ideologie des „Paramilitarismus“, Frankfurt a. M./Bern 1982.

Meinl, Susanne: „Hakenkreuz am Stahlhelm - Schwarz-weiß-rot das Band ...“. Friedrich Wilhelm Heinz und der Kampf gegen die Republik in Hessen 1920-1925, in: Zwischen Unruhe und Ordnung: ein deutsches Lesebuch für die Zeit von 1925-1960 am Beispiel einer Region: Mittelhessen, hrsg. von Gideon Schüler, Gießen 1989, S. 154-182.

Dies.: Ein konservativer Revolutionär in der Weimarer Republik und im „Dritten Reich“-eine politischbiographische Skizze des Friedrich Wilhelm Heinz 1918 bis 1945, Gießen (MS) 1990.

Dies./Krüger, Dieter: Der politische Weg von Friedrich Wilhelm Heinz. Vom Freikorpskämpfer zum Leiter des Nachrichtendienstes im Bundeskanzleramt, in: VfZ 42 (1994), S. 39-69.

Morsey, Rudolf: Die deutsche Zentrumspartei 1917-1923, Düsseldorf 1966.

Nagel, Irmela: Fememorde und Fememordprozesse in der Weimarer Republik, Diss. Köln 1991.

Neumann, Reinhard/Putlitz, Gisbert zu: Philipp Lenard 1862-1947, in: Semper Apertus. Sechshundert Jahre Ruprecht-Karls-Universität Heidelberg 1386-1986. Festschrift in sechs Bänden, Berlin/Heidelberg/New York u. a. 1985, Bd. III, S. 376-405.

Nobel, Alphons: Mord in der Politik, Hamburg/Berlin 1931.

Nußer, Horst G. W.: Konservative Wehrverbände in Bayern, Preußen und Österreich 1918-1933, München 1973.

Orth, Wilhelm: Walther Rathenau und der Geist von Rapallo. Größe und Grenzen eines deutschen Bürgers, Berlin (O) 1962.

Phelps, Reginald H.: „Before Hitler came“: Thule Society and Germanenorden, in: The Journal of Modern History 35 (1963), S. 245-261.

Pilhion, Roger: Ernst von Salomon. De l' Aventure à l' Écriture, Diss. Grenoble o. J. [1973].

Plat, Wolfgang: Attentate. Eine Sozialgeschichte des politischen Mordes, Düsseldorf/Wien 1982. 
Potthoff, Heinrich: Gewerkschaften und Politik zwischen Revolution und Inflation, Düsseldorf 1979.

Rasehorn, Theo: Rechtspolitik und Rechtssprechung. Ein Beitrag zur Ideologie der „Dritten Gewalt“, in: Karl Dietrich Bracher/Manfred Funke/Hans-Adolf Jacobsen (Hrsg.), Die Weimarer Republik 1918-1933. Politik - Wirtschaft - Gesellschaft, Bonn 1987, S. 407-428.

Reisberg, Arnold: Um die Einheitsfront nach dem Rathenaumord, in: Beiträge zur Geschichte der deutschen Arbeiterbewegung 5 (1963), S. 995-1009.

Rosenberg, Arthur: Entstehung der Weimarer Republik, hrsg. von Kurt Kersten, Frankfurt ${ }^{15} 1973$.

Rother, Bernd: Die Sozialdemokratie im Lande Braunschweig 1918 bis 1933, Bonn 1990.

Sabrow, Martin: Die Judenhetzerin von Kitzingen. Ein Lehrstück über den alltäglichen Antisemitismus in der Weimarer Zeit, in: Praxis Geschichte, 1992, H. 2, S. 59 ff.

Sauer, Wolfgang: Die Mobilmachung der Gewalt, Köln/Opladen 1974.

Schön, Eberhart: Dic Entstehung des Nationalsozialismus in Hessen, Meisenheim am Glan 1972.

Schüddekopf, Otto-Ernst: Nationalbolschewismus in Deutschland 1918-1933, Frankfurt a. M./Berlin/ Wien 1972.

Schulin, Ernst: Walther Rathenau. Repräsentant, Kritiker und Opfer seiner Zeit, Göttingen/Zürich/ Frankfurt a. M. 1979.

Ders.: Walther Rathenau und sein Integrationsversuch als „Deutscher jüdischen Stammes", in: Walter Grab (Hrsg.), Jüdische Integration und Identität in Deutschland und Österreich, Tel Aviv 1984, S. 13 38.

Ders.: Zu Rathenaus Hauptwerken, in: Schulin (Hrsg.), Rathenau. Hauptwerke und Gespräche, S. 499595.

Schultzendorff, Walther von: Proletarier und Prätorianer. Bürgerkriegssituationen aus der Frühzeit der Weimarer Republik, Köln 1966.

Schulz, Gerhard (Hrsg.): Weimarer Republik, Freiburg/Würzburg 1987.

Ders.: Zwischen Demokratie und Diktatur. Verfassungspolitik und Reichsreform in der Weimarer Republik, Bd. 1: Die Periode der Konsolidierung und der Revision des Bismarckschen Reichsaufbaus 1919-1930, Berlin/New York 21987.

Schulze, Hagen: Otto Braun oder Preußens demokratische Sendung. Eine Biographie, Frankfurt a. M./ Berlin/Wien 1977.

Ders.: Weimar. Deutschland 1917-1993, Berlin ${ }^{2} 1982$.

Schwend, Karl: Bayern zwischen Monarchie und Diktatur. Beiträge zur bayerischen Frage in der Zeit von 1918 bis 1933, München 1954.

Sinzheimer, Hugo/Fraenkel, Ernst: Die Justiz in der Weimarer Republik. Eine Chronik. Mit ciner Einleitung von Otto Kirchheimer, Neuwied/Bcrlin 1968.

Steffani, Winfried: Dic Untersuchungsausschüsse des Preußischen Landtages zur Zeit der Weimarer Republik. Ein Beitrag zur Entwicklung, Funktion und politischen Bedeutung parlamentarischer Untersuchungsausschüssc, Düsseldorf 1960.

Stern, Howard N.: Political Crime and Justice in the Weimar Republic, Diss. Baltimore 1966.

Ders.: The Organisation Consul, in: The Journal of Modern History 35 (1963), S. 20-32.

Streckfuß, Adolf: Berliner März. 1848, Bcrlin 1948.

Streit, Josef: Entwicklungstendenzen der Klassenjustiz in der Weimarer Republik, Diss. Berlin (O)1965.

Striesow, Jan: Die Dcutschnationale Volkspartei und die Völkisch-Radikalen 1918-1920, Bd. 1, Frankfurt a. M. 1981.

Theweleit, Klaus: Männerphantasien, 2 Bde., Reinbek bei Hamburg 1980.

Thoß, Bruno: Der Ludendorff-Kreis 1919-1923. München als Zentrum der mitteleuropäischen Gegenrevolution zwischen Revolution und Hitler-Putsch, München 1978.

Ders.: Nationale Rechtc, militärische Führung und Diktaturfrage in Deutschland 1913-1923, in: Militärgeschichtliche Mitteilungen 1987, H. 2, S. 27-76.

Venner, Dominique: Söldner ohne Sold. Die deutschen Freikorps 1918-1923, Wien o. J. [1974].

Volkov, Shulamit: Überlegungen zur Ermordung Rathenaus als symbolischem Akt, in: Buddensieg/ Hughcs/Kocka u. a., Ein Mann vieler Eigenschaften, S. 99-105.

Waite, Robert G. L.: Vanguard of Nazism. The Free Corps Movement in Postwar Germany 1918-1923, Cambridge/Mass. 1952.

Weller, B. Uwe: Maximilian Harden und die „Zukunft", Bremen 1970.

Werner, Andreas: SA und NSDAP. „Wehrverband“, Parteitruppe oder „Revolutionsarmee“? Studien zur Geschichte der SA und der NSDAP 1920-1933, Diss. Erlangen 1964.

Wette, Wolfram: Gustav Noskc. Eine politische Biographie, Düsseldorf 1987.

Wildc, Harry: Der politische Mord, Bayreuth 1962.

Ders.: Walther Rathenau in Selbstzeugnissen und Bilddokumenten, Reinbek bei Hamburg 1971. 
Williamson D.: Pro and contra Rathenau: The Controversy in the German Press 1914-1918, in: The Wiener Library Bulletin 28, 1975, S. 32-43.

Ders.: Walther Rathenau. A study of his political, industrial and cultural activities and of his reputation in contemporary Germany 1893 - June 1921, Diss. London 1971.

Winkler, Heinrich August: Die deutsche Gesellschaft der Weimarer Republik und der Antisemitismus, in: Bernd Martin/Ernst Schulin (Hrsg.), Die Juden als Minderheit in der Geschichte, München 1982, S. 271-289.

Ders.: Von der Revolution zur Stabilisierung. Arbeiter und Arbeiterbewegung in der Weimarer Republik 1918 bis 1924, Berlin/Bonn ${ }^{2} 1985$.

Ders.: Weimar 1918-1933. Die Geschichte der ersten deutschen Demokratie, München 1993.

Young, Harry F.: Maximilian Harden. Censor Germaniae. Ein Publizist im Widerstreit von 1892 bis 1927, Münster 1971.

Zorn, Wolfgang: Die politische Entwicklung des deutschen Studententums 1918-1931, in: Kurt Stephenson/Alexander Scharff/Wolfgang Klötzer (Hrsg.), Darstellungen und Quellen zur Geschichte der deutschen Einheitsbewegung im neunzehnten und zwanzigsten Jahrhundert, Bd. 5, Heidelberg 1965, S. 223-307. 


\title{
Abkürzungen
}

\author{
ADS Archiv der sozialen Demokratie \\ BA Bundesarchiv Koblenz \\ BA/P Bundesarchiv Koblenz, Abteilungen Potsdam \\ BayHStA Bayerisches Hauptstaatsarchiv \\ BavStA Bayerisches Staatsarchiv \\ BDC Berlin Document Center \\ CV Centralverein deutscher Staatsbürger jüdischen Glaubens \\ DLitM Deutsches Literaturarchiv Marbach \\ DVSTB Deutschvölkischer Schutz- und Trutzbund \\ EZB Evangelisches Zentralarchiv in Berlin \\ GStA Geheimes Staatsarchiv Preußischer Kulturbesitz \\ HStA Hauptstaatsarchiv \\ $\mathrm{HZ} \quad$ Historische Zeitschrift \\ IfZ Institut für Zeitgeschichte \\ LG Landgericht \\ Mdl Ministerium des Innern \\ MS Manuskript \\ NA National Archives and Records Administration \\ NL Nachlaß \\ NVS Nationalverband Deutscher Soldaten \\ ORA Oberreichsanwalt \\ PA/AA Politisches Archiv des Auswärtigen Amts \\ RA Rechtsanwalt \\ RMI Reichsminister des Innern \\ RMJ Reichsminister der Justiz \\ SAM Sonderarchiv Moskau \\ StAF Staatsarchiv Freiburg \\ StAH Staatsarchiv Hamburg \\ StAL Sächsisches Staatsarchiv Leipzig \\ VfZ Vierteljahrshefte für Zeitgeschichte \\ VNS Verband nationalgesinnter Soldaten \\ VO Verordnung \\ ZfG Zeitschrift für Geschichtswissenschaft
}




\section{Personenregister}

Verweise auf Anmerkungen sind kursiv gesetzt.

Abendroth, Friedrich von 12, 29

Abernon, Edgar Viscount d' 38

Ahrens, Georg 89

Alken (Untersuchungsrichter) 124

Alsberg, Max 104, 110

Andler, Rudolf 212

Andreae, Edith 89

Ankermann, Walter 171-183

Apelt (Polizeiwachtmeister) 86

Arndt, Ernst Moritz 199

Aronsfrau, Sina 139

Auer, Erhard 166

Baatz (Bankvorsteher) 177

Bade, Erich 12, 93, 96, 107, 151, 186, 187

Baldenius, Walter 30

Bang, Paul 17

Bauer, Max 21, 57, 170

Baur, Carl 184, 187, 190-195, 198, 207

Baur, Josef 193

Behrens, Erwin 93

Behrens, Till 93

Bismarck und Schönhausen, Otto von 117

Blei, Franz 83

Bloch, Paul 104, 174 f., 209, 213

Blome, Kurt 184-190, 193

Blumenfeld, Kurt 115

Bockmann, Richard 207

Böckel, Albert $31 \mathrm{f}$.

Boldt, Friedrich 129, 137

Bollinger, Wilhelm $30 \mathrm{f}$.

Borsig, Ernst von 73

Bose, von (Hauptmann) 126

Brammer, Karl 9

Brandt, Günther 93, 103, 106 f., 112-114, 130, $136,137-142,145$

Brandt, Karl 138

Braun, Otto 19,100

Breucker, Wilhelm 140 f., 157

Brockhusen, Eberhard von 47

Brïdigam, Theodor $12,63-67,84,90,110,121$, 125 ff., 133, 139, 154, 207

Bülow, Bernhard von 69

Burger, Alfred 26, 55

Büsch, Paul 98, 191-193, 194 f.

Calker, Wilhelm van 104

Claus, Felix 207

Cohn, Oskar 120, 151

Deutsch, Lili 82,85

Diestel, Arnold 162

Diestel, Franz 12, 92, 104, 107, 112, 115, 135, 162

Dietrich, Wolfgang 12, 102, 104, $130,138,196-$ 199
Diez, Karl 18

Dithmar, Kurt 129 ff., 133 f., 137 f., 197

Doehn, Bruno 104

Ebermayer, Ludwig 90, 103 ff., 109, 118, 135, 209, 211, $212 \mathrm{ff}$.

Ebert, Friedrich 20, 120, 150 f., 170

Edelmann, Wilhelm 66

Eggebrecht, Axel 8

Ehrentraut, Wilhelm 12, 30, 142, 146, 211

Ehrhardt, Hermann 9 f., 14 f., 21-25, 28- 44, 49$52,54,56,61,63,68,90,93,96,97,102,106 \mathrm{f}$., $109,113,116,120-128,130,136 \mathrm{f} ., 140,144,146$, 147-150, 153 ff., 157, 166, 169,178 ff., 183 f., $190,195,198-207,209,211$

Ehrler, Fritz 64, 66

Ellendt, Andrea 167

Erdmann, Karl-Dietrich 8

Erkens, Christina 119

Erzberger, Matthias $7 \mathrm{f} ., 10,17-28,49-56,62,64 f$, $67,69,81,90,105,111,118,122,126,128,134$ f., $146,149,165,168,170,177,200,206$

Escherich, Georg 43, 183

Federn-Kohlhaas, Etta 81

Fehrenbach, Konstantin 104

Fischer, Hermann 12, 92-103, 105-109, 111-114, $134,136-141,142,145-149,152,166,183-200$

Fischer, Wilma 189

Flesch, Fritz Werner 90

Fluth (Kriminalkommissar) 93, 186

Fraenkel, Paul 88

Freytag, Ernst $185 \mathrm{f}$.

Fritsch, Theodor 73

Fromholz, Hellmuth 182

Fürstenberg, Carl 72

Ganz, Hans 78

Gareis, Karl 7, 28, 105, 154

Gellert 12

Gerlach, Hellmut von 28, 70, 83 f., 121

Geßler, Otto 204

Goldschmidt, Alfons 73

Gollnick, Kurt 104

Göring, Hermann 203

Graefe, Albrecht von 168

Grenz, Albert Wilhelm 49, 171-183

Grünspach, Fritz 179

Gumbel, Emil Julius 9

Guderjahi, Max 91

Günther, Alfred $61 \mathrm{f}$.

Günther, Willi 12, 91-97, 104, 106-110, 112, 118, $135,140,146,147$

Haas, Ludwig 162

Haase, Hugo 7

Haefker, Walter $188 \mathrm{ff}$. 
Hänel, Rudolf $58,62 f$., 68,151

Hagens, Alfred 59, 104

Hahn, Gerhard 68

Hahn, Willy $104,111,213$

Haniel von Haimhausen, Edgar 89

Harden, Maximilian 8, 10 f., 28, 49, 69, 79, 105, $165,169-183$

Hartmann, Gustav 104

Haugwitz, Waldemar von 12

Heim, Georg 50

Heine, Heinrich 72

Heinrich Friedrich Karl, Prinz von Preußen 163

Heinz, Friedrich Wilhelm 10, 12, 15, 43, 59, 63, $65,84,90,111,117,120,125-128,130-134$, 138 f., 144, 150-154, 203

Heinze, Rudolf 12

Helfferich, Karl 17 f., 81, 90, 115, 163

Hellenbrecht, Kurt 207

Heller (Kriminalkommissar) 103, 196

Henning, Wilhelm 168

Henkel, Alfred 208

Henrich, Fritz Otro 30, 33

Hergt, Oskar 19

Herzig, Elisabeth 194

Hildebrandt, Friedrich 151, 186

Hildenbrand, Karl 104

Hiller, August 207

Himmler, Heinrich 50

Hindenburg, Paul von und Beneckendorff von 72,182

Hirschfeld, Oltwig von $17 \mathrm{f}$.

Hitler, Adolf 183, 202-205, 209

Hoegner, Wilhelm 41, 56

Hoelz, Max 57

Hoffmann, Alfred 12, $22 \mathrm{ff} ., 29-33,35 \mathrm{f}$., 38, $39 \mathrm{f}$., 41, 63-67, 90, 91, 105, 121-125, 136f., 140, 154, $157,188,203,204,207,208$ f., 210, 212, 214

Holländer, Ludwig 84

Holzweißig, Kurt 105

Hoppe, Karl 89

Houghton, Alanson 86

Hustert, Hans 13, 56-63, 67 f., 145, 150

Ilsemann, Christian 12, 93, 96, 104, 107, 112, 118, $135,136,152,187$

Jäckel, Hermann 104

Joël, Curt 212

Jünger, Ernst 115, 117

Kadow, Paul 185-188, 189, 190

Kagemann, Gustav 101

Kahl, Minna 185, 188

Kahr, Gustav Ritter von 21, 25, 43, 204 f.

Kaiser, Helene 88

Kapp, Wolfgang 15, 50, 29, 70, 126, 136, 149, 179, 205, 207

Karrenbrock, Lore 82
Kautter, Eberhard 29f., 33, 44, 131 f., 154, 202 f., 205, 209

Kern, Erwin 12, 14, 49, 59, 83, 92-103, 105-121, $128-135,166,183-200$

Kern, Gisela $101 \mathrm{ff} ., 118,130,137,138,144 f$., $148 f ., 166,189,198 f$.

Kern, Katharina 97

Kern, Walter 97

Kessler, Friedrich von 36

Kessler, Harry Graf 75, 82, 84 f., 87, 116, 143

Killinger, Manfred von 11, 12, 14, 18, 20, 22-27, $29,31 \mathrm{ff} ., 36,38,43,49,51-54,59,136,190,200$, 206, 209

Kittel, Rudolf 165

Klintzsch, Hans-Ulrich 30, 202, 203

Klotz, Helmut 153

Knilling, Eugen von 202

Koch, Erich 203

Kolb (SA-Mann) 203

Koppe, Ulrich 31

Korfanty, Wojcicch 31

Krebs, Alfred 137

Kriebel, Hermann 204

Krischbin, Walter $86 \mathrm{f}$.

Krull, Ernst 26, 48, 55

Küchenmeister, Fritz 93, 113

Küchenmeister, Johannes 12, 93, 98, 103, 106, 112 f., 138, 141, 145, 148

Kuhne, Paul 101

Kuhne, Walter 101

Lambach, Walther 73

Lampe, Heinrich 144, 148

Langenscheidt, Hans 185-188

Lauch, Herbert 33, 40, 42, 137, 144, 150

Lenard, Philipp $165 \mathrm{f}$.

Lcrchenfeld, Hugo Maximilian Graf von und zu 21, 168, 201

Lessing, Theodor 71

Liebe, Georg $152 \mathrm{f}$.

Liebe, Herbert 152

Liebknecht, Karl 26, 170

Liedig, Franz 67, 154

Link, Eduard 25

Lipinski, Richard 120, $150 \mathrm{ff}$.

Lossow, Otto Hermann von 202, 204

Löttgen (SA-Mann) 203

Loucheur, Louis 70

Ludendorff, Erich 10, 22, 36, 57, 70, 73 ff., 94, $140,155,170,179,183,205$

Luetgebrune, Walter $11,13,104,124,209,212 \mathrm{ff}$.

Lüttwitz, Walther Freiherr von 15, 50, 29, 70, 126, 136, 205

Luxemburg, Rosa 26

Malade, Theodor 103

Mamroth, Paul 92, 106

Mann, Thomas 157

Marckwald, H. 66 
Marx, Wilhelm 212

Meinecke, Fricdrich 157

Mesch, Lorenz 45, 49, 52 f.,181 f.

Mierendorff, Carlo 165

Mittag (Amtsgerichtsrat) 65

Möhl, Arnold Ritter von 201

Möhring, von (Rittmeister) 53

Müller, Adolf 25 f., 49

Müller, Heinrich 209

Müller, Herbert 24 f., 26 f.29 f., 33, 39, 190

Müller, Hermann (Reichskanzler) 104

Müller, Hermann (Reichstagsabgeordneter) 104

Müller von Hausen, Ludwig 48, $54 \mathrm{f}$.

Nernst, Walter 165

Niedner, Alexander $209 \mathrm{f}$.

Niedrig, Waldemar 12, $94 \mathrm{f} ., 104 \mathrm{ff} ., 109,111,118$, 135, 136-139, 146, 151 f., 169, 214

Niethammer, Emil 209 ff., 214

Noetzel, Theodor $62-66,84,90,127$

Nortz, Eduard 25 f.

Ochlschläger, Karl 13, 56-63, 67 f., 143, 145

Otto, Rudolf $184,190 \mathrm{ff}$.

Pabst, Waldemar 21

Parvus-Helphand, Alexander 48, 54 f., 116, 151

Peters, Ernst 166

Peters, Johannes 186, 189, 191, 192-195, 197

Pinner, Felix $77 \mathrm{f}$.

Pittinger, Otto 25, 44, 201, 205

Plaas, Hartmut 12, 15, 29, 30, 39, 40, 42f, 43, 44, 51 f., 59, 61 f., 64, 67, 84, 90, 93 ff., 104, 116- 119, $122 \mathrm{f} ., 126$ ff., 130, 134, 135, 138-142, 153 f., 195

Pöhner, Ernst $21 \mathrm{f}$., 35, 201

Pradelt, Heinrich 87

Prescher, Hans 182

Prince, Massow von 30

Prozeller, Josef $86,87 \mathrm{f}$.

Prüter, Willi $193 \mathrm{f}$.

Puttkamer, Franz von 98, 146, 186

Queiß, Georg von 36

Radbruch, Gustav 105, 109, 111, 162

Radek, Karl 114

Rathenau, Emil 48, 69

Rathenau, Mathilde 111, 162

Rathenau, Walther 7-10, 12-15, 28, 29, 48 f., $59 \mathrm{ff}$., 63-98,100,101, 102, 103-126, 128, 130, 132136, 138-158, 160, $161 \mathrm{ff} ., 165-170,177,189$, 191, 194-197, 200 f., 206 f., 211, 214 f.

Reinert (Hauptmann) 126, 127

Reinhardt, Walther 41, 133

Reventlow, Ernst Graf 76

Richter, H. J. 210

Richter, Wilhelm 89, 100

Ritter, Friedrich 184-187, 190, 191, 193

Roddie, Stewart 82

Röser, Karl 166
Rosenberg, Alfred 171

Roth, Albert 207

Roth, Alfred 77, 80

Roth, Christian 21, 22

Roth, Joseph 105

Ruge, Arnold 49, 83, 191

Sack, Alfons 104, 209, $213 f$.

Salomon, Ernst von $8 \mathrm{f} ., 12,15,28,38,40,51,59$, $87,89,95,104-107,109,111,112,117-120$, $125,128-136,138-142,144$ ff., 149-153, 157, 183, 195, 198, $198 \mathrm{ff.}$.

Scheel, Paul 98

Scheidemann, Philipp $7 \mathrm{f}$, $10 \mathrm{f} ., 13,20,35,41,56-$ $67,69,73,84,90,91,94,105,111,118,120 \mathrm{ff}$., $124,127,134,145,147,149-155,160,177,191$, 207,210

Schiff, Erich 174

Schiff, Victor 98

Schiffer, Eugen 73, 82

Schilchen, Marietta 209

Schmaltz, Elfride 178

Schmidt (Oberleutnant) 29 f., 127

Schmidt, Heinrich 59

Schmidt, gen. Schmidt- Halbschuh, Karl 14, 43, $51,56,111,131,150,152 \mathrm{ff}$.

Schmidt, Robert 75

Schneider, Otto (Bauarbeiter) 87

Schneider, Otto 153

Schoch, Karl Ritter von 163

Schroeder, Otto 128, 145, 153

Schücking, Walther 151

Schüder, Karl 30

Schulz, Heinrich 11, 22-27, 33 f., 37, 45, 49-55, 137,181

Schütt, Richard 12, 92, 104, 107 f., 110, 112, 115, $120,135,148$

Schweyer, Franz 21, 26, 101

Schwing, Ernst 130, 131, 132

Seeckt, Hans von 41

Seffner, Hans 40, 129

Seidl, Ludwig 45

Severing, Carl 11, 159, 203

Siebel, Karl 62, 129

Simon, Hugo-Ferdinand 83

Stauff, Philipp 47

Stein, Hans-Wilhelm 12, 101, 103, 104, 138, 196199

Steinbeck (SA-Mann) 203

Steinbeck, Gustav 12, 104, 112, 135, 136

Stinnes, Hugo 70, 77, 86, 158

Strassmann, Paul Ferdinand 88

Stubenrauch, Heinz 12, 92 f., 106, 109, 146

Stürtz, Emil 150

Sudermann, Hermann 89

Sundermeyer, Wilhelm 129

Techow, Ernst Werner 12, 15, 83, 91 ff., 96, 104115,117 f., 120 f., 122, 134, 135, 136, 137, 141, $142-148,150$ f., 187,214 
Techow, Gertrud 111, 120,143

Techow, Gustav 92

Techow, Hans-Gerd 12,15, $92 \mathrm{f} ., 95 \mathrm{f} ., 104,106 \mathrm{f}$., $112,117,135,136,142,146,150$

Thälmann, Ernst 94

Tillessen, Hcinrich 11, 22-27, 33, 34, 45, 49-55, $64,129,136,137,181$

Tillessen, Karl 12, 30, 61, 63-67, 84, 90, 91, 93 ff., 104, 115 f., 118, 121, 123, 125-144, 145, 150, 153 f., 195

Tillessen, Werner 93, 141

Tucholsky, Kurt 74, 81, 174, 179

Viebig, Erich 154

Völkel, Heinrich 89

Vornfett, August 34

Voß, Werner 104,112

Wagner, Eduard 163

Wagner, Erwin 130-135, 153

Warburg, Max 81, 150 ff., 154

Warnccke, Friedrich 12, 94, 95, 104, 106, 112, $135,136,151 \mathrm{f} ., 214$

Wartenberg, Ernst Graf Yorck von 182

Wassermann, Oskar 151

Wegelin, Johannes 129, 137, 203, 208

Wehrmann, Karl 62

Weichardt, Herbert 171-183

Weidemann, Philipp 131
Weismann, Robert 21, 25, 35 f., 40

Weiß, Bernhard 55, 82, 89, 92, 96, $97 \mathrm{ff}$.

Wels, Otto 91

Wende, Kurt 137 f., 144, 145, 190

Werber, Paul 30, 35, $38 f$., 62, 137, 145

Werthauer, Johannes 10,60

Wiese, Gottfried 191, 192-195

Wiese, Leopold von 73

Wiggers, Karl $186 f$., 193

Wilhelm I., König von Preußen und Deutscher Kaiser 162

Wilhelm II., Deutscher Kaiser und König von Preußen 71, 74, 80, 169

Wilson, Woodrow Theodor $169 \mathrm{f}$.

Winzer, Lothar 95

Wirth, Joseph 7, 19 ff., 43, 70, 76 f., 82-85, 116, $121,144,151,160,164,167,191,210$

Wissell, Rudolf 74

Wolff, Theodor $150 \mathrm{ff} ., 154$

Wulle, Reinhold 168

Zabel (Kriminalwachtmcistcr) 101

Zedlitz-Neukirch, Heinrich 31

Zeigner, Erich 120, 151

Zeiler, Alois 104

Zinn, Alexander 152

Zschauer, Max 207

Zwengauer, August 191

Zweig, Stefan 71,170 